\title{
OBSERVER DESIGN USING BOUNDARY INJECTIONS FOR PIPELINE MONITORING AND LEAK DETECTION
}

\author{
Ole Morten Aamo, Jørgen Salvesen, and Bjarne A. Foss
}

\author{
Department of Engineering Cybernetics \\ Norwegian University of Science and Technology \\ N-7491 Trondheim
}

\begin{abstract}
We design a leak detection system consisting of an adaptive Luenbergertype observer based on a set of two coupled one dimensional first order nonlinear hyperbolic partial differential equations governing the flow dynamics. It is assumed that measurements are only available at the inlet and outlet of the pipe, and output injection is applied in the form of boundary conditions. For the linearized model without friction and leak, exponential convergence of the state estimates to the plant state is shown by Lyapunovs method. The observer design is performed for the continuum model, ensuring that convergence properties established theoretically are not an artifact of the method of discretization. Heuristic update laws for adaptation of the friction coefficient and leak parameters are given, and simulations demonstrate their ability to detect, quantify and locate leaks.
\end{abstract}

Keywords: Partial differential equations; Observers; Adaptive systems; Pipeline leaks

\section{INTRODUCTION}

Transportation of liquids in pipelines requires monitoring to detect malfunctioning such as leaks. In the petroleum industry, leaks from pipelines may potentially cause environmental damage, as well as economic loss. These are motivating factors, along with requirements from environmental authorities, for developing efficient leak detection systems. While some leak detection methods are hardware-based, relying on physical equipment being installed along the pipeline, the focus of this paper is on software-based methods that work for cases with limited instrumentation. In fact, instrumentation in the petroleum industry is usually limited to the inlet and outlet of pipelines, only. This calls for sophisticated signal processing methods to obtain reliable detection of leaks. Some software-based leak detection methods perform statistical analysis on measurements (black box), while others incorporate models based on physical principles. Our method falls into the latter category, in that we will use a dynamic model of the pipe flow based on a set of two coupled hyperbolic partial differential equations.

There have been numerous studies on model based leak detection. We mention here the most relevant ones with regard to our work. Based on a discretized pipe flow model, Billman and Isermann (1987) designed an observer with friction adaptation. In the event of a leak, the outputs from the observer differs from the measurements, and this is exploited in a correlation technique that detects, quantifies and locates the leak. Verde (2001) used a bank of observers, computed by the method for fault detection and isolation developed by Hou and Müller (1994). The underlying model is a linearized, discretized pipe flow model on a grid of $N$ nodes. The observers are designed in 
such a way that all but one will react to a leak. Which one of the $N$ observers that does not react to the leak depends on the position of the leak, and this is the mechanism by which the leak is located. The outputs of the remaining observers are used for quantifying the leak. The bank of observers are computed using the recursive numerical procedure suggested by Hou and Müller (1994), however it was shown in Salvesen (2005) that due to the simple structure of the discretized model, the observers may be written explicitly. This is important, because it removes the need for recomputing the bank of observers when the operating point of the pipeline is changed. Verde (2004) also proposed a nonlinear version, using an extremely coarse discretization grid.

Several companies offer commercial solutions to pipeline monitoring with leak detection. Fantoft (2005) uses a transient model approach in conjunction with the commercial pipeline simulator OLGA2000, while EFA Technologies (1987, 1990, 1991) uses an event detection method that looks for signatures of no-leak to leak transitions in the measurements.

The detection method of Verde (2001) using a bank of observers, can potentially detect multiple leaks. However, multiple simultaneous leaks is an unlikely event, so the complex structure of a bank of $N$ observers seems unnecessary. In this paper, we instead employ ideas from adaptive control, treating the size and location of a single point leak as constant unknown parameters. This differs from the method of Billman and Isermann (1987), since we will model the leak in the observer, thereby obtaining state estimates also in a leak situation. Another important aspect of our method is that the observer is designed for the continuum model, ensuring that convergence properties established theoretically are not an artifact of the method of discretization. Our leak detection system consists of an adaptive Luenbergertype observer, based on a set of two coupled one dimensional first order nonlinear hyperbolic partial differential equations governing the flow dynamics. It is assumed that measurements are only available at the inlet and outlet of the pipe, and output injection is applied in the form of boundary conditions. Heuristic update laws for adaptation of the friction coefficient and the two leak parameters are suggested.

\section{MATHEMATICAL MODEL}

\subsection{Physical Model}

For liquid flow in a pipe we have the mass conservation

$$
\frac{\partial p}{\partial t}+u \frac{\partial p}{\partial x}+\rho c^{2} \frac{\partial u}{\partial x}=0
$$

and the momentum conservation (ignoring friction for now)

$$
\frac{\partial u}{\partial t}+u \frac{\partial u}{\partial x}+\frac{1}{\rho} \frac{\partial p}{\partial x}=0
$$

for $(x, t) \in(0, L) \times(0, \infty)$, and where $u$ is flow velocity, $p$ is pressure, and $\rho$ is density. The relation between pressure and density is modelled as (Nieckele et al. (2001))

$$
\rho=\rho_{\text {ref }}+\frac{p-p_{\text {ref }}}{c^{2}},
$$

where $\rho_{\text {ref }}$ is a reference density at reference pressure $p_{\text {ref }}$, and $c$ is the speed of sound. Equation (1)-(2) also describes gas flow in a pipe, simply by replacing (3) with the ideal gas law. Under the conditions we consider, we assume $c$ is sufficiently large to ensure $\rho>0$. In compact form, we have

$$
\frac{\partial}{\partial t}\left[\begin{array}{l}
p \\
u
\end{array}\right]+A(p, u) \frac{\partial}{\partial x}\left[\begin{array}{l}
p \\
u
\end{array}\right]=0
$$

where, using (3),

$$
\begin{aligned}
A(p, u) & =\left[\begin{array}{cc}
u & k+p \\
\frac{c^{2}}{k+p} & u
\end{array}\right], \\
k & =c^{2} \rho_{\text {ref }}-p_{\text {ref }},
\end{aligned}
$$

and the boundary conditions are

$$
\begin{gathered}
u(0, t)=u_{0}(t), \\
p(L, t)=p_{L}(t) .
\end{gathered}
$$

The eigenvalues of $A$ are

$$
\lambda_{1}=u-c, \lambda_{2}=u+c .
$$

Assuming that $u \ll c$, which is always the case in the applications we are considering, the eigenvalues are distinct and satisfy

$$
\lambda_{1}<0<\lambda_{2} .
$$

The system is therefore strictly hyperbolic. Steady state solutions $(\bar{p}, \bar{u})$ of $(4)$, must satisfy

$$
A(p, u) \frac{\partial}{\partial x}\left[\begin{array}{l}
p \\
u
\end{array}\right]=0 .
$$

Since $A(p, u)$ is invertible $\left(\lambda_{i} \neq 0\right)$, we have that

$$
\frac{\partial \bar{p}}{\partial x}=0, \frac{\partial \bar{u}}{\partial x}=0,
$$

so $\bar{p}$ and $\bar{u}$ are constant. The boundary conditions (7)-(8) yield $\bar{p}=p_{L}$ and $\bar{u}=u_{0}$.

\subsection{Model in Characteristic Form}

Consider now the change of coordinates

$$
\alpha(p, u)=c \ln \left(\frac{k+p}{k+\bar{p}}\right)+u-\bar{u},
$$




$$
\beta(p, u)=-c \ln \left(\frac{k+p}{k+\bar{p}}\right)+u-\bar{u},
$$

which clearly is defined for all physically feasible $p$ and $u$. It is easy to see that it's inverse is

$$
\begin{aligned}
& p(\alpha, \beta)=(k+\bar{p}) \exp ((\alpha-\beta) /(2 c))-k \\
& u(\alpha, \beta)=\bar{u}+(\alpha+\beta) / 2
\end{aligned}
$$

Notice that the fixed point $(\bar{p}, \bar{u})$ corresponds to $(0,0)$ in the new coordinates. The time derivative of (13)-(14) is

$$
\begin{aligned}
& \alpha_{t}=c \frac{p_{t}}{k+p}+u_{t}, \\
& \beta_{t}=-c \frac{p_{t}}{k+p}+u_{t} .
\end{aligned}
$$

Inserting for $p_{t}$ and $u_{t}$ from (4) yields

$$
\begin{aligned}
\alpha_{t} & =-c \frac{u p_{x}+(k+p) u_{x}}{k+p}-\left(\frac{c^{2}}{k+p} p_{x}+u u_{x}\right) \\
& =-(u+c)\left(c \frac{p_{x}}{k+p}+u_{x}\right) \\
& =-(u+c) \alpha_{x} \\
\beta_{t} & =c \frac{u p_{x}+(k+p) u_{x}}{k+p}-\left(\frac{c^{2}}{k+p} p_{x}+u u_{x}\right) \\
& =-(u-c)\left(-c \frac{p_{x}}{k+p}+u_{x}\right) \\
& =-(u-c) \beta_{x} .
\end{aligned}
$$

Using (16), we obtain

$$
\begin{aligned}
& \alpha_{t}+(\bar{u}+c+(\alpha+\beta) / 2) \alpha_{x}=0, \\
& \beta_{t}+(\bar{u}-c+(\alpha+\beta) / 2) \beta_{x}=0 .
\end{aligned}
$$

The boundary conditions are obtained from (15)(16), and are

$$
\begin{aligned}
\alpha(0, t)+\beta(0, t) & =0, \\
\alpha(L, t)-\beta(L, t) & =0 .
\end{aligned}
$$

The characteristic form (21)-(22) is convenient for the observer design carried out in the next section.

\section{OBSERVER DESIGN}

In reality, input signals to pipelines are usually choke openings at the inlet and outlet. Here, we instead view $u_{0}(t)$ and $p_{L}(t)$ in $(7)-(8)$ as inputs to the process, and construct the copy of the plant dynamics (4)

$$
\frac{\partial}{\partial t}\left[\begin{array}{l}
\hat{p} \\
\hat{u}
\end{array}\right]+A(\hat{p}, \hat{u}) \frac{\partial}{\partial x}\left[\begin{array}{l}
\hat{p} \\
\hat{u}
\end{array}\right]=0,
$$

with boundary conditions

$$
\hat{u}(0, t)=u_{0}(t),
$$

$$
\hat{p}(L, t)=p_{L}(t) .
$$

Notice that the input to (4)-(8) is also copied in (25)-(27). Equation (25)-(27) can be viewed as a Luenberger-type observer, and convergence is guaranteed when the process is operated at asymptotically stable fixed points, which is usually the case for pipelines. However, we look for alternatives to the boundary conditions (26)-(27) which yield better convergence properties. Taking $p_{0}(t)=p(0, t)$ and $u_{L}(t)=u(L, t)$ as process measurements, we may apply output injection to (25)-(27). In transformed coordinates, we obtain

$$
\begin{aligned}
& \hat{\alpha}_{t}+(\bar{u}+c+(\hat{\alpha}+\hat{\beta}) / 2) \hat{\alpha}_{x}=0, \\
& \hat{\beta}_{t}+(\bar{u}-c+(\hat{\alpha}+\hat{\beta}) / 2) \hat{\beta}_{x}=0
\end{aligned}
$$

with boundary conditions (we omit the argument $t$ for brevity)

$$
\begin{aligned}
\hat{\alpha}(0)+\hat{\beta}(0) & =b_{0}(\alpha(0), \beta(0), \hat{\alpha}(0), \hat{\beta}(0), \\
& \alpha(L), \beta(L), \hat{\alpha}(L), \hat{\beta}(L)), \\
\hat{\alpha}(L)-\hat{\beta}(L) & =b_{L}(\alpha(0), \beta(0), \hat{\alpha}(0), \hat{\beta}(0), \\
& \alpha(L), \beta(L), \hat{\alpha}(L), \hat{\beta}(L)),
\end{aligned}
$$

where $b_{0}$ and $b_{L}$ are functions to be designed. Notice that the boundary injections (30)-(31) may be any function of the known signals at both ends of the pipe. For convergence analysis, we consider the linearization of (21)-(22) and (28)(29) around $(0,0)$ and form the dynamics of the observer error, defined as $\tilde{\alpha}=\alpha-\hat{\alpha}, \tilde{\beta}=\beta-\hat{\beta}$. We obtain

$$
\frac{\partial}{\partial t}\left[\begin{array}{c}
\tilde{\alpha} \\
\tilde{\beta}
\end{array}\right]+\Lambda \frac{\partial}{\partial x}\left[\begin{array}{c}
\tilde{\alpha} \\
\tilde{\beta}
\end{array}\right]=0
$$

with boundary conditions

$$
\begin{aligned}
\tilde{\alpha}(0)+\tilde{\beta}(0) & =-b_{0}(\alpha(0), \beta(0), \hat{\alpha}(0), \hat{\beta}(0), \\
\alpha(L), \beta(L), \hat{\alpha}(L), \hat{\beta}(L)), & (33) \\
\tilde{\alpha}(L)-\tilde{\beta}(L) & =-b_{L}(\alpha(0), \beta(0), \hat{\alpha}(0), \hat{\beta}(0), \\
\alpha(L), \beta(L), \hat{\alpha}(L), \hat{\beta}(L)), & (34)
\end{aligned}
$$

where

$$
\Lambda=\left[\begin{array}{cc}
\bar{u}+c & 0 \\
0 & \bar{u}-c
\end{array}\right] .
$$

Following $\mathrm{Xu}$ and Sallet (2002) and Coron et al. (2004), consider the Lyapunov function candidate

$$
\begin{aligned}
V=\frac{1}{c+\bar{u}} \int_{0}^{L} & \tilde{\alpha}^{2} e^{-\mu x /(c+\bar{u})} d x \\
& +\frac{1}{c-\bar{u}} \int_{0}^{L} \tilde{\beta}^{2} e^{\mu x /(c-\bar{u})} d x,
\end{aligned}
$$


recalling the assumption that $c \gg|\bar{u}|$. The time derivative of $V$ along solutions of (32)-(34) is

$$
\begin{aligned}
\dot{V} & =-\mu V+\tilde{\alpha}^{2}(0)-\tilde{\beta}^{2}(0) \\
& -\tilde{\alpha}^{2}(L) e^{-\mu L /(c+\bar{u})}+\tilde{\beta}^{2}(L) e^{\mu L /(c-\bar{u})} .
\end{aligned}
$$

At this point we need to select $b_{0}$ and $b_{L}$ such that $\tilde{\alpha}^{2}(0)-\tilde{\beta}^{2}(0)$ and $-\tilde{\alpha}^{2}(L) e^{-\mu L /(c+\bar{u})}+$ $\tilde{\beta}^{2}(L) e^{\mu L /(c-\bar{u})}$ are negative. We adopt the particularly simple choice made in Coron et al. (2004), and select $b_{0}$ and $b_{L}$ such that

$$
\begin{gathered}
\tilde{\alpha}(0)=k_{0} \tilde{\beta}(0), \\
\tilde{\beta}(L)=k_{L} \tilde{\alpha}(L),
\end{gathered}
$$

in which case

$$
\begin{aligned}
\dot{V} & =-\mu V-\left(1-k_{0}^{2}\right) \tilde{\beta}^{2}(0) \\
& -\left(e^{-\mu L /(c+\bar{u})}-k_{L}^{2} e^{\mu L /(c-\bar{u})}\right) \tilde{\alpha}^{2}(L) .
\end{aligned}
$$

So, if

$$
\left|k_{0}\right| \leq 1 \text { and }\left|k_{L}\right| \leq e^{-\mu L / c}
$$

then

$$
\dot{V} \leq-\mu V
$$

Since (36) defines a norm equivalent to the $L_{2}$ norm on $[0, L]$, it follows that system (32) with (38)-(39) is exponentially stable at the origin in the $L_{2}$ norm. Notice that (41) implies that whenever $\left|k_{L}\right|<1$, there exists $\mu>0$ for which (42) holds. Replacing (26)-(27) with the new boundary conditions, the observer becomes

$$
\begin{gathered}
\frac{\partial \hat{p}}{\partial t}+\hat{u} \frac{\partial \hat{p}}{\partial x}+(k+\hat{p}) \frac{\partial \hat{u}}{\partial x}=0 \\
\frac{\partial \hat{u}}{\partial t}+\frac{c}{k+\hat{p}} \frac{\partial \hat{p}}{\partial x}+\hat{u} \frac{\partial \hat{u}}{\partial x}=0
\end{gathered}
$$

with boundary conditions

$$
\begin{aligned}
& \hat{u}(0)=u(0)+c \frac{1-k_{0}}{1+k_{0}} \ln \left(\frac{k+p(0)}{k+\hat{p}(0)}\right), \\
& \hat{p}(L)=(k+p(L)) \\
& \quad \times \exp \left(\frac{k_{L}-1}{c\left(1+k_{L}\right)}(u(L)-\hat{u}(L))\right)-k .
\end{aligned}
$$

When $k_{0}=1$ and $k_{L}=1,(45)-(46)$ reduces to (26)-(27). It is interesting to notice that the above Lyapunov analysis does not provide exponential convergence in this case. Another interesting observation to make is that the design is independent of the working condition $(\bar{u}, \bar{p})$. Figure 1 shows the observer error in terms of evolution in time of the $L_{2}(0, L)$ norm of $u(x, t)-\hat{u}(x, t)$ for the cases with and without output injection (the $L_{2}$ norm of $p(x, t)-\hat{p}(x, t)$ looks qualitatively the same).
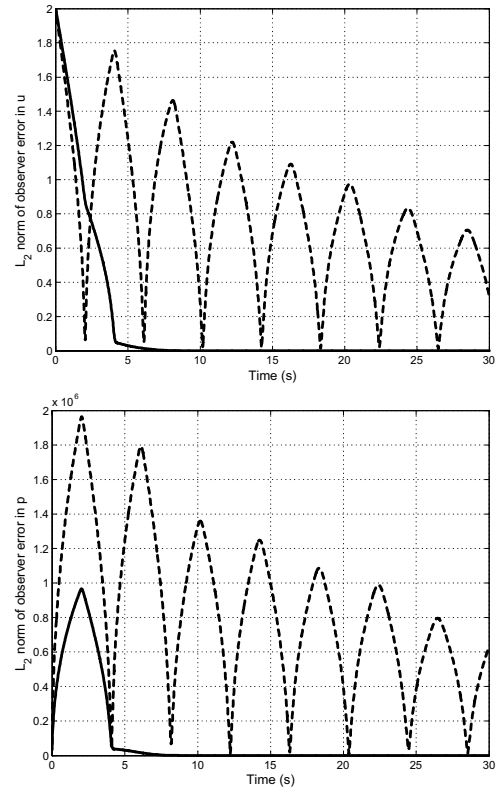

Fig. 1. Observer error with (solid) and without (dashed) output injection.

\section{ADAPTATION OF FRICTION COEFFICIENT}

Adding friction to the model (4), we have the mass balance

$$
\frac{\partial p}{\partial t}+u \frac{\partial p}{\partial x}+(k+p) \frac{\partial u}{\partial x}=0
$$

and momentum conservation

$$
\frac{\partial u}{\partial t}+u \frac{\partial u}{\partial x}+\frac{c^{2}}{k+p} \frac{\partial p}{\partial x}=-(1+\Delta) \frac{f}{2} \frac{|u| u}{D},
$$

where $D$ is the pipe diameter, and $\Delta$ is considered an unknown constant that accounts for uncertainty in the friction coefficient $f$, which is given by Schetz and Fuhs (1996)

$$
\frac{1}{\sqrt{f}}=-1.8 \log _{10}\left[\left(\frac{\epsilon / D}{3.7}\right)^{1.11}+\frac{6.9}{\operatorname{Re}_{d}}\right] .
$$

$\epsilon / D$ is the pipe relative roughness, $\operatorname{Re}_{d}$ is the Reynolds number defined as

$$
\operatorname{Re}_{d}=\frac{\rho u D}{\mu},
$$

and $\mu$ is the fluid viscosity. The observer is then

$$
\begin{gathered}
\frac{\partial \hat{p}}{\partial t}+\hat{u} \frac{\partial \hat{p}}{\partial x}+(k+\hat{p}) \frac{\partial \hat{u}}{\partial x}=0 \\
\frac{\partial \hat{u}}{\partial t}+\hat{u} \frac{\partial \hat{u}}{\partial x}+\frac{c^{2}}{k+\hat{p}} \frac{\partial \hat{p}}{\partial x}=-(1+\hat{\Delta}) \frac{\hat{f}}{2} \frac{|\hat{u}| \hat{u}}{D}
\end{gathered}
$$

which incorporates an estimate $\hat{\Delta}$ of $\Delta$, and with boundary conditions (45)-(46). Consider the heuristic parameter update law

$$
\dot{\hat{\Delta}}=-\kappa_{\Delta}(\tilde{\alpha}(L)+\tilde{\beta}(0)),
$$

where $\kappa_{\Delta}$ is a strictly positive constant. In physical coordinates, equation (53) corresponds to

$$
\dot{\hat{\Delta}}=-\kappa_{\Delta}\left(\varphi_{1}+\varphi_{2}\right) \text {, }
$$




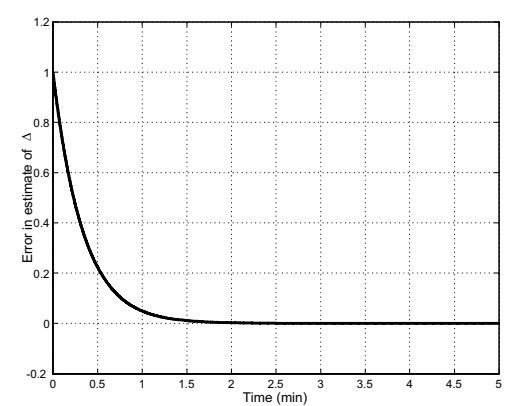

Fig. 2. Error in estimated friction factor, that is $\Delta-\hat{\Delta}$.

where

$$
\begin{aligned}
& \varphi_{1}=u(0)-\hat{u}(0)+c \ln \left(\frac{k+\hat{p}(0)}{k+p(0)}\right), \\
& \varphi_{2}=u(L)-\hat{u}(L)+c \ln \left(\frac{k+p(L)}{k+\hat{p}(L)}\right) .
\end{aligned}
$$

Figure 2 shows the evolution of $\Delta-\hat{\Delta}$ when the initial friction in the observer is twice that of the plant.

\section{LEAK DETECTION}

Adding a leak to the model (47)-(48), with $\Delta=0$, we have the mass balance

$$
\frac{\partial p}{\partial t}+u \frac{\partial p}{\partial x}+(k+p) \frac{\partial u}{\partial x}=-\frac{c^{2}}{A} f_{l}(x),
$$

and the momentum conservation

$\frac{\partial u}{\partial t}+u \frac{\partial u}{\partial x}+\frac{c^{2}}{k+p} \frac{\partial p}{\partial x}=-\frac{f}{2} \frac{|u| u}{D}+\frac{1}{A} \frac{c^{2}}{k+p} u f_{l}(x)$,

where $A$ is the pipe cross sectional area. Assuming a point leak, we select $f_{l}(x)$ as

$$
f_{l}(x)=w_{l} \delta\left(x-x_{l}\right),
$$

where $w_{l}$ and $x_{l}$ are the size of the leak and position of the leak, respectively, and $\delta$ denotes the Dirac distribution. The observer is then

$$
\begin{aligned}
\frac{\partial \hat{p}}{\partial t}+\hat{u} \frac{\partial \hat{p}}{\partial x}+(k+\hat{p}) \frac{\partial \hat{u}}{\partial x}=-\frac{c^{2}}{A} \hat{w}_{l} \delta\left(x-\hat{x}_{l}\right), \\
\frac{\partial \hat{u}}{\partial t}+\hat{u} \frac{\partial \hat{u}}{\partial x}+\frac{c^{2}}{k+\hat{p}} \frac{\partial \hat{p}}{\partial x} \\
=-\frac{\hat{f}}{2} \frac{\hat{u} \mid \hat{u}}{D}+\frac{1}{A} \frac{c^{2}}{k+\hat{p}} \hat{u} \hat{w}_{l} \delta\left(x-\hat{x}_{l}\right),
\end{aligned}
$$

which incorporates estimates of the leak size and position, $\hat{w}_{l}, \hat{x}_{l}$. Consider the heuristic parameter update laws

$$
\dot{\hat{w}}_{l}=-\kappa_{w}(\tilde{\beta}(0)-\tilde{\alpha}(L)),
$$

and

$$
\dot{\hat{x}}_{l}=-\kappa_{x} \varphi_{\alpha \beta}\left|\varphi_{\alpha \beta}\right|^{\frac{1}{\gamma}-1},
$$

where

$$
\varphi_{\alpha \beta}=\tilde{\alpha}(L)+\tilde{\beta}(0),
$$

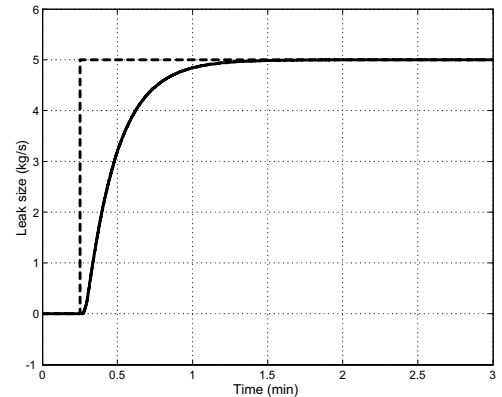

Fig. 3. Estimated size of leak (solid) and actual size of leak (dashed).

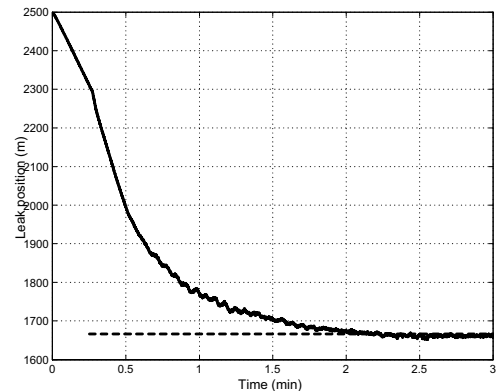

Fig. 4. Estimated position of leak (solid) and actual position of leak (dashed).

and $\kappa_{w}, \kappa_{x}$ and $\gamma$ are strictly positive constants. In physical coordinates, equation (62)-(63) corresponds to

$$
\begin{aligned}
& \dot{\hat{w}}_{l}=-\kappa_{w}\left(\varphi_{1}-\varphi_{2}\right) \\
& \dot{\hat{x}}_{l}=-\kappa_{x}\left(\varphi_{1}+\varphi_{2}\right)\left|\varphi_{1}+\varphi_{2}\right|^{\frac{1}{\gamma}-1},
\end{aligned}
$$

where $\varphi_{1}$ and $\varphi_{2}$ are given in (55)-(56). Figures $3-4$ show the evolution of the estimates (65)-(66) for a leak occuring at $t=0.25$ minutes.

\section{SIMULATIONS WITH OLGA}

The leak detection test in the previous section was a nominal test, where the plant dynamics and the observer dynamics were identical (except for output injection, of course). Here, we perform more realistic tests, replacing the plant dynamics by the state-of-the-art flow simulator OLGA2000 ${ }^{1}$. For two different cases, summarized in Table 1, we run our leak detection scheme (60)-(63) with (45)(46). In the table, $w_{\text {in }}$ denotes the mass rate of fluid at the inlet. Figure 5 shows that the leaks are quantified very accurately, while localization is somewhat noisy. However, the average error in position taken over the last 15 seconds shown in the Figure, is within $0.25 \%$ and $0.36 \%$ of the pipe length for Cases I and II, respectively.

1 OLGA2000 is a commercially available flow simulator widely used by the petroleum industry. It is developed by Scandpower AS. 


\begin{tabular}{c|c|c|c}
\hline Parameter & Case I & Case II & Unit \\
\hline$L$ & 990 & 8000 & $\mathrm{~m}$ \\
\hline$D$ & 0.10 & 0.51 & $\mathrm{~m}$ \\
\hline$c$ & $1.26 e 3$ & $1.2 e 3$ & $\mathrm{~m} / \mathrm{s}$ \\
\hline$k$ & $1.38 e 9$ & $1.24 e 9$ & $\mathrm{~Pa}$ \\
\hline$\mu$ & 0.0063 & 0.0056 & $\mathrm{~Pa} \mathrm{~s}$ \\
\hline$\epsilon$ & 0 & 0 & $\mathrm{~m}$ \\
\hline$\hat{\Delta}$ & 0.13 & 0.028 & - \\
\hline$w_{l}$ & 4.9 & 12.5 & $\mathrm{~kg} / \mathrm{s}$ \\
\hline$x_{l}$ & 505 & 4000 & $\mathrm{~m}$ \\
\hline$w_{i n}$ & 70 & 300 & $\mathrm{~kg} / \mathrm{s}$ \\
\hline
\end{tabular}

Table 1. Numerical coefficients.
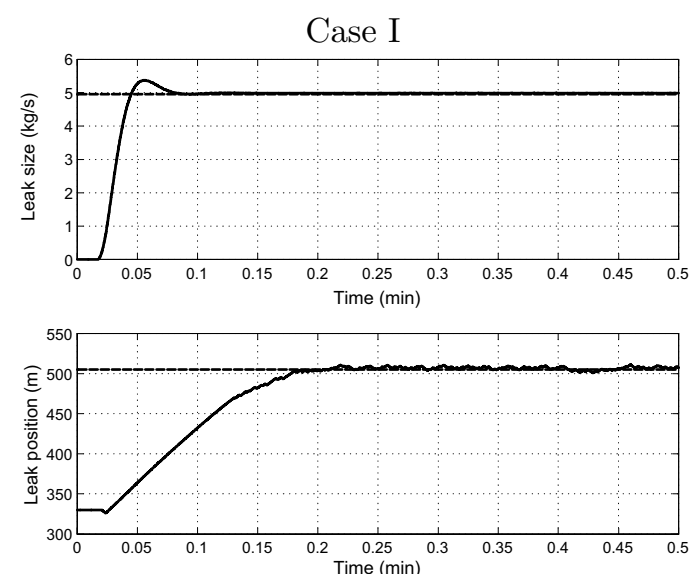

Case II
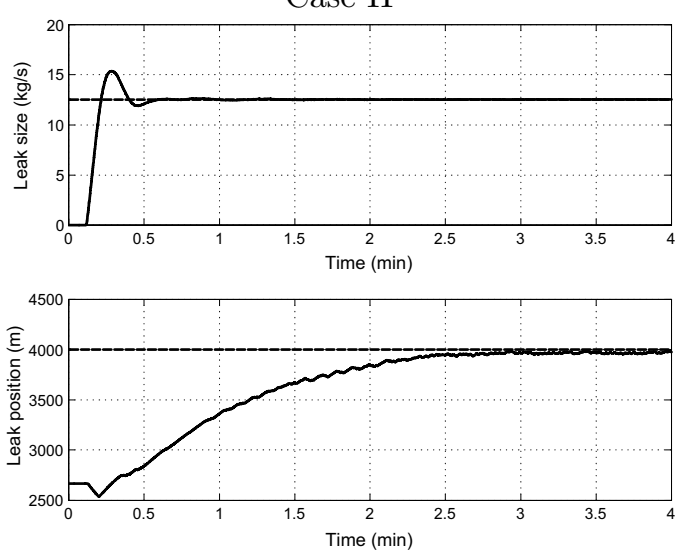

Fig. 5. Leak detection applied to OLGA simulations.

\section{CONCLUDING REMARKS}

We have designed a leak detection system for pipelines consisting of an adaptive Luenbergertype observer and heuristic update laws for the parameters characterizing a point leak. The only available process information is flow velocity and pressure at the inlet and outlet of the pipe. Simulations with a state-of-the-art flow simulator as process, demonstrate accurate quantification and localization in two test cases.

\section{ACKNOWLEDGEMENTS}

We gratefully acknowledge the support from the Gas Technology Center at NTNU, Statoil, and the Norwegian Research Council.

\section{REFERENCES}

L. Billmann and R. Isermann, "Leak detection methods for pipelines," Automatica, vol. 23, no. 3, pp. 381-385, 1987.

J.-M. Coron, B. d'Andrea-Novel, and G. Bastin, "A strict Lyapunov function for boundary control of hyperbolic systems of conservation laws," Proceedings of the 43rd IEEE Conference on Decision and Control, Paradise Island, Bahamas, December 14-17, 2004.

EFA Technologies, Inc., "Events at a leak," Technical paper available from EFA on request, 1987.

EFA Technologies, Inc., "Effect of pressure measurement resolution on PPA leak detection," Technical paper available from EFA on request, 1990.

EFA Technologies, Inc., "PPA event suppression capability," Technical paper available from EFA on request, 1991.

Fantoft, "D-SPICE overview: Production management systems," Fantoft Process Technologies, 2005.

J.-M. Greenberg and T.-t. Li, "The effect of boundary damping for the quasilinear wave equations," Journal of Differential Equations, vol. 52, pp. 66-75, 1984.

J. de Halleux, C. Prieur, J.-M. Coron, B. d'Andrea-Novel, and G. Bastin, "Boundary feedback control in networks of open channels," Automatica, vol. 39, pp. 1365-1376, 2003.

M. Hou and P. Müller, "Fault detection and isolation observers," International Journal of Control, vol. 60, no. 5, pp. 827-846, 1994.

A.O. Nieckele, A.M.B. Brage, and L.F.A. Azevedo, "Transient pig motion through gas and liquid pipelines," Journal of Energy Resources Technology, vol. 123, no. 4, 2001.

J. Salvesen, Leak Detection by Estimation in an Oil Pipeline, MSc Thesis, NTNU, 2005.

J.A. Schetz and A.E. Fuhs, Handbook of Fluid Dynamics and Fluid Machinery, Volume 1, Fundamentals of Fluid Mechanics, John Wiley \& Sons, 1996.

D.N. Shields, S.A. Ashton, and S. Daley, "Design of nonlinear observers for detecting faults in hydraulic sub-sea pipelines," Control Engineering Practice, vol. 9, pp. 297-311, 2001.

C. Verde, "Multi-leak detection and isolation in fluid pipelines," Control Engineering Practice, vol. 9, pp. 673-682, 2001.

C. Verde, "Minimal order nonlinear observer for leak detection," Journal of Dynamic Systems, Measurement and Control, vol. 126, pp. 467$472,2004$.

C.-Z. Xu and G. Sallet, "Exponential stability and transfer functions of processes governed by symmetric hyperbolic systems," ESAIM Journal on Control, Optimisation and Calculus of Variations, vol. 7, pp. 421-442, 2002. 